\title{
Equipos de salud y su funcionalidad: oportunidades para el tratamiento del tabaquismo
}

\author{
JAIME SAPAG M.*, MÓNICA ACUÑA M.**, ALEX CAMPOS A.***, ÁNGEL BENÍTEZ V.****, RAÚL VI- \\ LLARROEL P.*****, LEONARDO VÉJAR M.****** y MARÍA PAZ CORVALÁN*******
}

\section{Health teams and their functionality: opportunities for smoking cessation}

This chapter proposes actions for an adequate implementation of the strategies defined in the Guidelines of Clinical Practice for Tobacco Treatment, Chile 2017. In the public subsystem, significant and progressive efforts have been made to carry out smoking detection and counseling $A B C$ on the various devices in the network. In the private subsystem on the other hand specialized services have been carried out: Intensive Therapy for Smoking Cessation. A deficit of integration of services is detected based on clear and shared goals that allow to reach a relevant population impact. Guidelines are proposed to be followed to achieve success in the interventions for the treatment of smokers.

Key words: Smoking; smoking cessation; tobacco, counseling.

\section{Resumen}

Este capitulo propone acciones para una adecuada implementación de las estrategias definidas en las Primeras Guías de Práctica Clínica del Tratamiento del Tabaquismo, Chile 2017. En el subsistema público por una parte se han hecho esfuerzos importantes y progresivos para realizar la detección de fumadores y la consejería breve ABC en los distintos dispositivos de la red. En el subsistema privado por otra parte se han llevado adelante servicios especializados: Terapia Intensiva de Cesación del Tabaquismo. Se detecta un déficit de integración de servicios a partir de metas claras y compartidas que permitan alcanzar un impacto poblacional relevante. Se proponen lineamientos a seguir para lograr éxito en las intervenciones para el tratamiento de los fumadores.

Palabras clave: Fumar; dejar de fumar; tabaco, consejería.

El objetivo de este artículo es plantear una propuesta para la adecuada implementación de las estrategias definidas en las Guías de Práctica Clínica de Cesación del Tabaquismo, 2017, en el sistema de salud chileno, considerando tanto el sector público como al privado, desde una perspectiva de red integral.

\section{Situación Actual}

Es importante reconocer la experiencia ya existente de incorporación de servicios para personas con consumo de tabaco en nuestro país ${ }^{1,2,3,4,5,6,7,8}$.

Iniciativas interesantes se han incorporado, por ejemplo, en las Salas de Infecciones Respiratorias

\footnotetext{
* Dptos. de Salud Pública y Medicina Familiar, Escuela de Medicina, Facultad de Medicina, Pontificia Universidad Católica de Chile. \& Project Scientist, Office of Transformative Global Health, Institute for Mental Health Policy Research, Centre for Addiction and Mental Health, Ontario, Canada.

** $\quad$ Enfermera. Coordinadora Programa de Tabaco Clínica Santa María.

*** Kinesiólogo, Encargado Programa ERA. CESFAM Alberto Allende Jones, Talagante.

**** Kinesiólogo, Especialista en Kinesiología Respiratoria, Programa ERA, CESFAM Cerro Navia.

***** Kinesiólogo, Programa ERA CESFAM Dr. Lucas de la Sierra.

****** Pediatra Especialista Broncopulmonar, Experto en Tabaquismo SEPAR, Programa Tabaquismo Servicio de Salud Metropolitano Sur Oriente, Chile.

******* Programa de Tabaquismo Centro Médico Fundación del Banco Estado, Coordinadora Comisión Tabaco, Sociedad Chilena de Enfermedades Respiratorias.
} 
Agudas (IRA) y de Enfermedades Respiratorias Agudas (ERA), Odontología y Pesquisa de Tabaquismo, Programa de Consejerías Breves, Sistema de Detección Precoz, Intervención Breve y Referencia a Tratamiento (SBIRT) ${ }^{9}$, así como en la articulación con estrategias asociadas a detección e intervención breve respecto a alcohol y a otras sustancias psicoactivas. Igualmente, se reconocen algunos avances en cuanto a capacitación en el tema liderados por el Ministerio de Salud. El subsistema privado, por otra parte, ha llevado adelante servicios especializados (ej. "clínicas de tabaco"), pero con muy baja cobertura y que habitualmente conllevan copagos importantes para los usuarios, limitando el acceso.

Se detecta un déficit en la definición e integración de metas claras y compartidas que permitan alcanzar un impacto poblacional relevante. Es urgente contar con una estrategia integral, de mediano y largo plazo, para responder a este problema. Es de particular relevancia, considerar los aspectos críticos para avanzar ${ }^{10}$, según se conceptualiza en las denominadas Ciencias de la Implementación ${ }^{11}$.

\section{Propuesta}

Considerando la necesidad de abordar esta brecha, se plantea un trabajo escalonado y priorizando lo siguiente, en base a la evidencia ${ }^{12,13,14,15}$ y a la realidad del contexto nacional:

Se propone una estrategia multi-componente, teniendo en cuenta experiencia internacional en este campo ${ }^{3,16,17,18}$ y el contexto local, que incluya - al menos - lo siguiente:

- Aumentar a 10\%, la tasa de consejo breve tipo ABC-D mínimo, realizable en 1 minuto en las consultas habituales de APS (atención primaria) por parte de todos los profesionales priorizados en personas con enfermedades crónicas no transmisibles. Se visualiza un rol particularmente relevante en los profesionales de enfermería y kinesiología.

- Realizar tratamiento intensivo de tabaquismo en los consultorios externos de todos los hospitales base del país, en todos los centros APS de más de 100.000 inscritos. Reforzar supervisión de hogares libres de humo de tabaco de los pacientes crónicos en control.

- Ofrecer ABC-D asociado a Tratamiento Intensivo del Tabaquismo a todos los pacientes hospitalizados especialmente por problemas cardiovasculares, respiratorios, diabetes $\mathrm{y}$ cáncer. Indispensable en todas las unidades coronarias del país.

(1) -Consejería telefónica: Implementar línea
800 para su acceso sin $\operatorname{costo}^{19}$ : A través de Salud Responde. Difundir y usar para derivar hospitalizados y el seguimiento en APS, COSAM (Centro de Salud Mental) y alta de hospitalización. Se Puede hacer seguimiento haciendo uso del sistema informático (SISTAB).

\section{Actividades a desarrollar}

- Incorporar tabaquismo a los objetivos estratégicos de la salud.

- Iniciar jornadas de información /capacitación con todos los directivos de salud ministeriales y comunales.

- Desarrollar capacitación necesaria para los diferentes niveles de intervención, centros hospitalarios y centros APS.

- Incorporar medicamentos de cesación del tabaco en los arsenales básicos de Centros de Atención Primaria y Secundaria.

- Incorporar Programa Tabaquismo al GES de Programas EPOC, Cardiovascular, Diabetes, Cáncer y de Salud Mental.

Para implementar adecuadamente lo anterior, se sugiere:

- Mesa de Trabajo con Ministerio de Salud para priorizar y definir mejores vías de implementación.

- Evaluación económica de alternativas y planificación detallada.

- Sensibilización y motivación a equipos locales/incluyendo a directivos.

- Definición de coordinación y equipo que participará en la gestión de la estrategia en cada dispositivo asistencial. Ello implica destinar y programar recursos para la adecuada implementación.

- Diagnósticos locales respecto a situación de tabaquismo y respuestas implementadas, de manera de identificar brechas y oportunidades para implementar estrategias pertinentes.

- Entrenamiento al Equipo de Salud ${ }^{20}$. Se propone capacitación interprofesional en distintos niveles, incluyendo un Curso Intensivo o Diplomado para quienes liderarán la estrategia en los distintos Centros, de modo que ellos también capaciten al equipo del establecimiento. Además, una capacitación inicial vía "on-line" para la mayor parte del equipo de salud, por ejemplo, usando el Sistema de Aprendizaje a Distancia -SIADdel MINSAL (Formación en Intervenciones de Cesación del Consumo de Tabaco). Se plantea la necesidad de acreditar estos procesos para que se consideren en la carrera funcionaria. 
- Recordatorios que promuevan el Consejo Breve o ABC-D en personas fumadoras. (habitualmente, como parte del sistema informático).

- Disponibilidad de medicamentos para tratamiento de la adicción a la nicotina (acceso, gratuidad...).

- Difusión de información a la comunidad sobre la estrategia.

- Supervisión y feedback local.

- Definición de metas específicas y realistas por establecimiento, redes, Servicios de Salud, país.

- Incentivos: metas asociadas a entrega de prestaciones de cesación del tabaco, por ejemplo, consejería breve.

- Sistema de registros e información y adecuado monitoreo y evaluación continua.

Nota final:

- La discusión sobre los recursos necesarios para implementar esta propuesta es muy importante y estratégica.

\section{Bibliografía}

1.- MINSAL. (2013). Guía Clínica AUGE: Enfermedad Pulmonar Obstructiva Crónica - EPOC- (Segunda Edición). Disponible en: http://www.bibliotecaminsal.cl/ wp/wp-content/uploads/2016/04/Enfermedad-PulmonarObstructiva-Cr\%C3\%B3nica.pdf

2.- MINSAL \& OPS/OMS. (2003). La cesación del consumo de tabaco: Manual para el Equipo de Salud. Disponible en: https://www.chilelibredetabaco.cl/descargas/ centrodoc/Manual_cesacion_MinSal.pdf

3.- ALCÁNTARA-GÓMEZ J, MARTÍNEZ-GUTIÉRREZ J, BAMBS C, et al. (2016). Consejo breve sobre el tabaco en atención primaria de salud en Chile: un desafio pendiente. ARS MEDICA Revista de Ciencias Médicas, 41: 14-22.

4.- VEJAR L. Cómo los doctores pueden ayudar a disminuir el daño a la salud causado por el cigarrillo. Neumología Pediátrica 2011; 6: 3-7.

5.- VÉJAR L, MEDINA G, PONCE R, et al. (2013). Tratamiento de tabaquismo en funcionarios de la salud en 11 centros de Atención Primaria. Prev Tab, 15: 21-5.

6.- BELLO S. Tratamiento del Tabaquismo. Rev. Chil Cardiol 2011; 30: 230-9.

7.- SEIJAS D. Tabaquismo. Rev. Med. Clin. Condes 2008; 19: 99-113.

8.- ARAYA A, LEAL S, HUERTA G, FERNÁNDEZ A, FERNÁNDEZ O, MILLONES G. Consumo de tabaco y uso del consejo médico estructurado como estrategia preventiva del tabaquismo en médicos chilenos. Revista Médica de Chile 2012; 140: 347-52.

9.- POBLETE F, BARTICEVIC NA, ZUZULIC MS, PORTILLA R, CASTILLO-CARNIGLIA A, SA-
PAG JC, et al. A randomized controlled trial of a brief intervention for alcohol and drugs linked to the Alcohol, Smoking and Substance Involvement Screening Test (ASSIST) in primary health care in Chile. Addiction 2017; 112: 1462-9.

10.- WEST R, RAW M, MCNEILL A, STEAD L, AVEYARD P, BITTON J, et al. Health-care interventions to promote and assist tobacco cessation: a review of efficacy, effectiveness and affordability for use in national guideline development. Addiction 2015; 110: 1388-403.

11.- LOBB R, GRAHAM A. Implementation Science and its Application to Population Health. Annu Rev Public Health 2013; 34: 235-51.

12.- HARTMANN-BOYCE J, STEAD LF, CAHILL K, LANCASTER T. Efficacy of interventions to combat tobacco addiction: Cochrane update of 2012 reviews. Addiction 2013; 108: 1711-21.

13.- USPSTF. Counseling and Interventions to Prevent Tobacco Use and Tobacco-Caused Disease in Adults and Pregnant Women: U.S. Preventive Services Task Force Reaffirmation Recommendation Statement. Ann Inter Med 2009;150: 551-5.

14.- New Zealand Government. The New Zealand Guidelines for Helping People to Stop Smoking, ed. Ministry of Health. 2014.

15.- STEAD LF, BUITRAGO D, PRECIADO N, SANCHEZ G, HARTMANN-BOYCE J, LANCASTER T. (2013). Physician advice for smoking cessation. The Cochrane Database of Systematic Reviews, CD000165.

16.- MARTÍN CANTERA C, PUIGDOMÈNECH E, BALLVÉ JL, ARIAS OL, CLEMENTE L, CASAS $\mathrm{R}$, et al. Effectiveness of multicomponent interventions in primary healthcare settings to promote continuous smoking cessation in adults: a systematic review. BMJ Open. British Medical Journal Publishing Group 2015, 5(10):e008807-16.

17.- PAPADAKIS S, MCDONALD P, MULLEN K-A, REID R, SKULSKY K, PIPE A. Strategies to increase the delivery of smoking cessation treatments in primary care settings: a systematic review and meta-analysis. Prev Med 2010; 51: 199-213.

18.- PAPADAKIS S, PIPE A, KELLY S, PRITCHARD G, WELLS GA. (2015) Strategies to improve the delivery of tobacco use treatment in primary care practice. The Cochrane Database of Systematic Reviews, CD011556.

19.- STEAD LF, HARTMANN-BOYCE J, PERERA R \& LANCASTER T. (2013). Telephone counselling for smoking cessation. The Cochrane Database of Systematic Reviews, CD002850.

20.- CARSON KV, VERBIEST ME, CRONE MR, BRINN MP, ESTERMAN AJ, ASSENDELFT WJ, et al. (2012). Training health professionals in smoking cessation. The Cochrane Database of Systematic Reviews, CD000214.

Correspondencia a:

Dr. Jaime Sapag M.

Email: jsapag@med.puc.cl 